\title{
Ultrasonographic assessment of the extent of hepatic steatosis in severe malnutrition
}

\author{
J F Doherty, E J Adam, G E Griffin, M H N Golden
}

Tropical Metabolism

Research Unit,

University of the

West Indies,

Kingston,

Jamaica

J F Doherty*

M H N Golden $†$

St George's Hospital

Medical School,

London,

Department of

Communicable Diseases

J F Doherty:

G E Griffin

Department of Radiology

E J Adam

Department of Medicine and Therapeutics, University of Aberdeen M H N Goldent

Correspondence to: Dr M H N Golden, Department of Medicine and Therapeutics, University of Aberdeen, Foresterhill,

Aberdeen AB9 2ZD.

Accepted 8 July 1992

\begin{abstract}
Ultrasonographic, blinded assessment was made of the extent of hepatic steatosis in $\mathbf{5 5}$ children with severe malnutrition: undernutrition $(n=6)$, marasmus $(n=18)$, marasmickwashiorkor $(n=17)$, and kwashiorkor $(n=14)$. The children were examined on admission, in early recovery (considered as baseline), and again at discharge. Eleven healthy control children and eight of the previously malnourished children were studied as comparison groups.

Both oedematous and non-oedematous malnourished children had significantly more steatosis than the comparison groups at each time. Children with oedematous malnutrition had significantly greater steatosis than nonoedematous children at admission. Half of the non-oedematous malnourished children had appreciable hepatic steatosis at both admission and at baseline.
\end{abstract}

Hepatic fat was only slowly mobilised. The rate constant was $1.4 \pm 0 \cdot 3 \% /$ day. One quarter of the children did not change steatosis grades during the period they were in hospital. There was no overall correlation between the extent of steatosis and liver size.

Hepatic steatosis in childhood malnutrition is not confined to oedematous children: it is frequently present in marasmic and undernourished children. Its extent is not necessarily related to the degree of hepatomegaly and accumulated lipid is only slowly mobilised.

\section{(Arch Dis Child 1992;67:1348-52)}

Kwashiorkor, or oedematous childhood malnutrition, was described by Cecily Williams in 1935. Her description included the statement: 'Post-mortem, the only constant finding is an extreme fatty infiltration of the liver'. 1 The precise pathogenesis of this accumulation of fat in the liver remains unexplained; however, the severity of the pathological change is beyond doubt. The median fat content of liver biopsy samples taken from severely malnourished Jamaican children was $30-35 \%$, and it appro- ached $50 \%$ of liver wet weight in the severest cases. ${ }^{2}$ Whatever the pathogenesis, disturbances of liver function, such as increases in bilirubin ${ }^{3}$ and serum transaminases, ${ }^{4}$ carry a very bad prognosis.

Hepatic steatosis is usually held to be a clinical feature only of oedematous malnutrition ${ }^{5}$ and standard textbooks categorically state that 'fatty liver does not occur in marasmus'. ${ }^{6}$ Waterlow, however, mentions one marasmic child with a fat content of $35 \%$ liver wet weight ${ }^{2}$ and other authors have reported similar cases. ${ }^{7-9}$ It is unclear if these are isolated cases or whether hepatic steatosis frequently occurs in marasmus and undernutrition-the nonoedematous forms of childhood malnutrition.

Previous studies have used chemical analysis or histological grading of liver biopsy tissue to assess liver steatosis. These series were, of necessity, limited to patients with definite indications for biopsy and therefore excluded both children with very severe illness and those with mild forms of the disease; further, in very few patients has more than a single assessment been made.

Recently ultrasonography has been validated and used to assess liver fatty infiltration in adults; the hepatic steatosis can be demonstrated with high sensitivity (94\%) and specificity $(84 \%){ }^{10}$

The aim of the present study was to examine the extent of hepatic steatosis, longitudinally, in an unselected series of severely malnourished Jamaican children.

\section{Subjects and methods}

STUDY CHILDREN

The study group comprised 55 children, 6 to 36 months old. They were consecutively admitted to the Tropical Metabolism Research Unit in Kingston, Jamaica, as a result of severe, primary malnutrition. A summary of their clinical details and diagnoses, using the Wellcome classification, ${ }^{11}$ is given in table 1 . The children were treated by standard protocols. ${ }^{12}$ Their diet was based on an infant formula (Pelargon, Nestlé)

Table 1 The clinical details of the study children; values are mean (SD)

\begin{tabular}{lllll}
\hline & $\begin{array}{l}\text { Undermutrition } \\
(n=6)\end{array}$ & $\begin{array}{l}\text { Marasmus } \\
(n=18)\end{array}$ & $\begin{array}{l}\text { Marasmic-kwashiorkor } \\
(n=17)\end{array}$ & $\begin{array}{l}\text { Kwashiorkor } \\
(n=14)\end{array}$ \\
\hline Male:female & $5: 1$ & $13: 5$ & $11: 6$ & $9: 5$ \\
Age (months) & $18 \cdot 6(4 \cdot 7)$ & $16 \cdot 7(6 \cdot 8)$ & $12 \cdot 3(6 \cdot 3)$ & $12 \cdot 8(3 \cdot 1)$ \\
Admission height for age (Z) & $-2 \cdot 7(1 \cdot 3)$ & $-4 \cdot 2(1 \cdot 6)$ & $-4 \cdot 2(1 \cdot 2)$ & $-2 \cdot 1(1 \cdot 0)$ \\
Admission weight for height (Z) & $-2 \cdot 5(1 \cdot 1)$ & $-3 \cdot 0(0 \cdot 7)$ & $-2 \cdot 2(1 \cdot 0)$ & $-1 \cdot 6(0 \cdot 9)$ \\
Discharge weight for height (Z) & $+0 \cdot 1(0 \cdot 6)$ & $-0 \cdot 6(0 \cdot 7)$ & $+0 \cdot 1(0 \cdot 5)$ & $+0 \cdot 1(0 \cdot 6)$ \\
Time admission to baseline study (days) & $11(3)$ & $8(3)$ & $9(2)$ & $13(6)$ \\
Time baseline to discharge study (days) & $29(10)$ & $34(10)$ & $26(11)$ & $26(7)$ \\
\hline
\end{tabular}

$\mathrm{Z}=\mathrm{SD}$ scores from the NCHS standard height of a child of the same age and weight for a child of the same height. 
fortified with vegetable oil (corn or coconut) to give an energy density of $5 \cdot 7 \mathrm{MJ} / \mathrm{kg}$, vitamins, and minerals. ${ }^{13}$

\section{COMPARISON GROUPS}

Eleven children who were resident in a local children's home were studied as a control group. Eight previously malnourished children, who had had grade 0 or 1 steatosis at discharge, were also studied between 32 and 162 days after discharge, as a further comparison group. Each of these children was assessed as clinically healthy; they were anthropometrically within 1 SD of the median weight of a child of the same height and sex (National Center for Health Statistics (NCHS) standards).

\section{CONSENT}

Informed consent was obtained from each child's parent or guardian. The study was approved by the ethics committees of the University of the West Indies and of the Ministry of Health, Jamaica.

\section{ULTRASOUND PROCEDURE}

Each study child had ultrasonography performed on three occasions:

(1) Within 48 hours of admission (admission);

(2) As soon as the acute signs of malnutrition had resolved but the child had not yet gained any appreciable weight (baseline). This was defined as the first point in time at which the children were oedema free and had gained weight at a rate of $5 \mathrm{~g} / \mathrm{kg} / \mathrm{day}$, for three consecutive days.

(3) Once the child had regained weight to within 1 SD of the median weight of a child of the same sex and height (NCHS standards) and had spontaneously reduced its appetite so that it was gaining weight at a normal rate (discharge).

Control children were studied once.

On each occasion, hepatic ultrasonography was performed using an Ultramark IV machine (Advanced Technology Laboratories) with a 5 $\mathrm{MHz}$ probe, by the same investigator (JFD). Photographic prints were obtained at each study; these were randomly coded, shuffled, and sent to England. The extent of hepatic steatosis was assessed from the coded prints alone, independently and blindly with respect to the child's diagnosis and the stage of recovery at which the study was performed, by a single assessor (EJA). The assessment was based on previously published criteria, ${ }^{10}$ modified to provide a numerical severity score. This ranged from $0=$ normal to $5=$ very severe. Mild steatosis was recognised by a slight increase in liver echogenicity and a slight exaggeration of the echo discrepancy between liver and kidney (grade 1). Moderate steatosis was accompanied by loss of echoes from the walls of the portal veins, resulting in a featureless appearance, with a degree of posterior beam attenuation and a greater discrepancy between the liver and kidney echo pattern (grade 3). Very severe steatosis was determined by a still greater degree of posterior beam attenuation, loss of echoes from most of the portal vein, and a marked discrepancy between liver and kidney (grade 5) ${ }^{10}$; grades 2 and 4 had intermediate appearances.

Assessment of liver size was made by measuring the liver span, in the mid-clavicular line, using ultrasonographically determined surface markers. This measurement was standardised by expressing liver span as a percentage of body length. Span was not recorded in the control children.

\section{STATISTICAL ANALYSIS}

Statistical analysis was performed using the Systat Software package (Systat Inc). Nonparametric statistics (Kruskal-Wallis and Friedman analysis of variance (ANOVA) and Mann-Whitney tests) were used for comparison of the degree of hepatic steatosis, as the severity score is not a continuous variable. Parametric statistics (ANOVA) were used for analysis of the liver span data after testing for normality.

A smoothing algorithm ${ }^{14}$ was used to assess the rate of disappearance of liver fat graphically; the program fits a line through a set of points using distance weighted least squares quadratic regression. A tension of $0 \cdot 1$ was used. The resulting lines are similar to those produced by the method of Cleveland. ${ }^{15}$

\section{Results}

STUDY CHILDREN

Children without oedema (undernutrition and marasmus) were older than the oedematous (marasmic-kwashiorkor and kwashiorkor) children $(p<0.05)$, and a higher proportion were male (table 1 ). The undernourished children were not as stunted as the marasmic children: however, they were just as wasted (deficit in weight-for-height); this indicates that the two non-oedematous groups had had similar recent nutritional insults. The times between the three studies were not different among the groups.

All three assessments were made on 51 of the 55 children: one child was considered too ill for ultrasonography to be performed at admission; two children died (one with marasmickwashiorkor after the admission, the other, with kwashiorkor, after the admission and baseline study); one child was inadvertently discharged before the final ultrasound.

\section{HEPATIC STEATOSIS}

The results are shown in fig 1 . Control subjects, with one exception, had a steatosis grade of 0 or 1. The exception was a 16 month old boy, resident in the children's home, who was assessed as having grade 2 steatosis. Clinical examination of the child and conventional liver function tests were normal. The previously malnourished children, studied at follow up also had either grade 0 or grade 1 steatosis.

At admission 51 of the 54 children had evidence of fat in their livers: the one child who was too ill for assessment, at this time, had grade 5 steatosis at baseline. For each of the diagnoses, at both admission and baseline, the extent of 


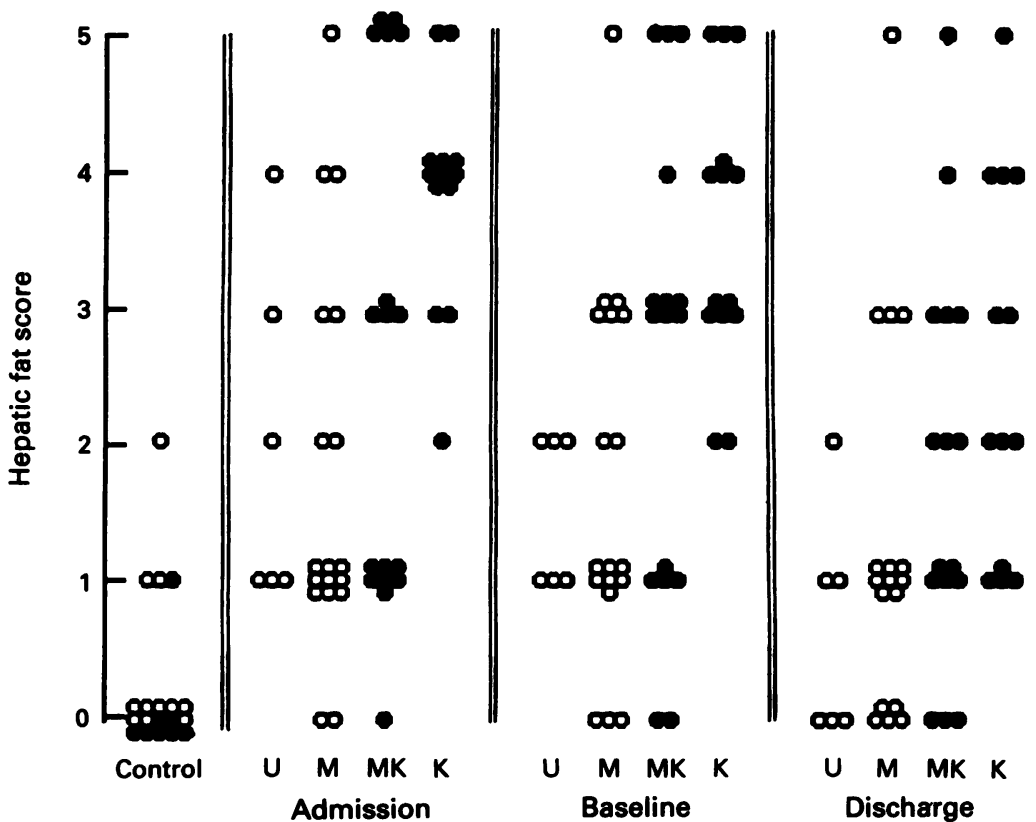

Figure 1 The hepatic fat score in control and previously malnourished children and in groups of malnourished children on admission, after resuscitation but before any appreciable weight gain (baseline), and at discharge. The control open dots are for children from a children's home, the control closed dots are for previously malnourished children. For the malnourished groups: $U$, undernutrition; $M$, marasmus; $M K$, marasmic-kwashiorkor; $K$, kwashiorkor; open dots, non-oedematous malnutrition; closed dots, oedematous malnutrition. Each dot represents a separate subject.

hepatic steatosis was significantly greater than the controls (fig 1$)(p<0.01)$. The extent of hepatic steatosis was different within the four groups, at each stage of the study $(p<0.02)$. The grade was greater in children with oedematous malnutrition than non-oedematous and greater in those with kwashiorkor than those with marasmic-kwashiokor $(p<0.0001)$. Children without oedema, however, had appreciable hepatic steatosis (fig 1). Eight of 17 children with marasmus and three of six with undernutrition had an hepatic ultrasound severity score $\geqslant 2$ both at admission and at baseline.

RECOVERY OF FATTY LIVER

Figure 2 shows the distance weighted least

Table 2 Rate of mobilisation of liver fat during recovery from malnutrition; values are mean (SEM)

\begin{tabular}{lll}
\hline Group & $\begin{array}{l}\text { No } \\
\text { in group }\end{array}$ & $\begin{array}{l}\text { Rate constant (/day) } \\
\text { baseline to discharge }\end{array}$ \\
\hline Undernutrition & 3 & 0.013 \\
Marasmus & 12 & $0.008(0.004)$ \\
Marasmic-kwashiorkor & 11 & $0.019(0.007)$ \\
Kwashiorkor & 13 & $0.016(0.005)$ \\
Non-oedematous & 15 & $0.009(0.004)$ \\
Oedematous & 24 & $0.017(0.004)$ \\
All patients & 39 & $0.014(0.003)$ \\
\hline
\end{tabular}

None of the groups were significantly different.

Table 3 Liver size* in malnourished children; values are mean (SD)

\begin{tabular}{lllll}
\hline & $\begin{array}{l}\text { Undermutrition } \\
(n=6)\end{array}$ & $\begin{array}{l}\text { Marasmus } \\
(n=18)\end{array}$ & $\begin{array}{l}\text { Marasmic-kwashiorkor } \\
(n=17)\end{array}$ & $\begin{array}{l}\text { Kwashiorkor } \\
(n=14)\end{array}$ \\
\hline Admission & $8 \cdot 1(2 \cdot 3)$ & $8 \cdot 1(1 \cdot 3)$ & $8 \cdot 9(2 \cdot 1)$ & $9 \cdot 5(1 \cdot 8)$ \\
Baseline & $8 \cdot 2(0 \cdot 9)$ & $7 \cdot 8(1 \cdot 0)$ & $8 \cdot 5(1 \cdot 7)$ & $9 \cdot 2(1 \cdot 1)$ \\
Discharge & $9 \cdot 2(1 \cdot 2)$ & $7.6(1 \cdot 3)$ & $8 \cdot 3(1 \cdot 1)$ & $8 \cdot 3(1 \cdot 0)$ \\
\hline
\end{tabular}

${ }^{*}$ Liver size measured as liver span $(\mathrm{cm}) \times 100 /$ height of child $(\mathrm{cm})$.

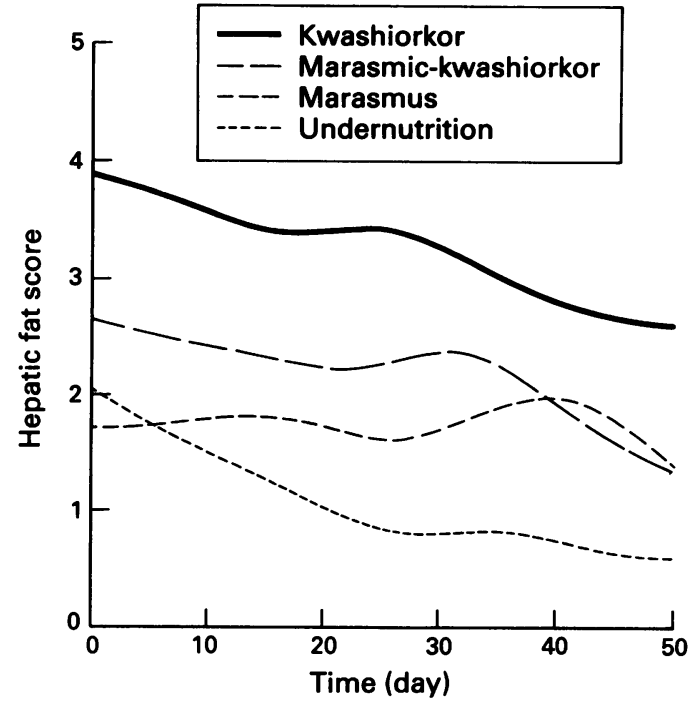

Figure 2 The distance weighted least squares quadratic regression lines of the hepatic fat score in the four groups of malnourished children with time.

squares quadratic regression lines of the hepatic steatosis grade, with time from admission, by diagnosis. Over the time of observation there was a gradual reduction in the fat score, so that by discharge there were significantly lower scores $(p<0.01)$ in each category (fig 1). However, only three of 29 children with an admission diagnosis of oedematous malnutrition and eight of 22 with previous non-oedematous malnutrition had attained a score of 0 by discharge. Furthermore, about one quarter of both the oedematous (seven of 29) and nonoedematous children (five of 22) had the same score $(\geqslant 2)$ at baseline and at discharge; during rehabilitation, these children had not mobilised sufficient fat to alter the abnormal ultrasonographic appearance of their livers. If the intervals between the severity grades are approximately equal and the mobilisation of fat is exponential, ${ }^{2}$ then a rate constant $(K)$ for mobilisation of liver fat can be calculated for each of the children that had a score of greater than $\mathbf{0}$ at discharge. These constants, calculated between baseline and discharge, are shown in table 2 . Overall only $1.4 \%$ of the liver fat was mobilised each day. There were no statistical differences between the rate constants of any of the diagnostic categories.

\section{LIVER SPAN}

The liver span was about $1 \mathrm{~cm}$ larger in oedematous than non-oedematous children at both admission and baseline; these differences were significant (table 3 ). There was no significant effect of time of study on the liver span and no interaction between time and diagnosis. Overall, there was no relationship between the degree of hepatic steatosis and the size of the liver. When the different time points were analysed separately there was a weak positive correlation between the liver span and the degree of hepatic steatosis at admission (Spearman's rho $=0.308, p<0.05$ ); the relationship was not significant at baseline or discharge. 


\section{Discussion}

The precise pathogenesis of fatty liver is unknown. Several theories to explain its occurrence in kwashiorkor have been proposed: endocrine abnormalities, ${ }^{16}$ increased fat synthesis, ${ }^{8}$ redistribution from adipose tissue, ${ }^{17}$ reduced lipoprotein synthesis, ${ }^{18} 19$ abnormalities of lipoprotein lipase ${ }^{20}$ and peroxisomal dysfunction, ${ }^{21}$ have each been put forward as playing a part. If the child recovers the fat disappears apparently without any long term sequelae. $^{22}$

The extent of liver fat in the present population was not measured directly; however, the cases were similar to those studied by Waterlow in whom the median amount of fat was about $30-35 \%$ of total liver wet weight. ${ }^{2}$ The distribution of his cases in \% fat classes and of our cases into grades was also similar. Thus, we would anticipate our grade 4 to correspond to Waterlow's $30-40 \%$ fat and our grade 5 to more than $40 \%$ fat. Although this is an assumed correspondence between the grades and chemical composition, the extent of liver fat in these children is likely to be much greater than in the adult population in which the method was quantitatively validated. Nevertheless, our assessment was made in a masked fashion by an experienced ultrasonographer who had no knowledge of the subjects.

The study confirms the frequency of fatty liver in children with oedematous malnutrition, although there are a few oedematous children without appreciable fatty liver (3-6\%). However, our results also show that fatty liver is usually present in marasmus and undernutrition-nonoedematous forms of malnutrition. In the Wellcome classification of malnutrition, ${ }^{11}$ nutritional oedema is equated with kwashiorkor; however, fatty liver is part of the clinical syndrome of kwashiorkor. ${ }^{1}$ The finding, that fatty liver is frequently present in marasmus, blurs the differences between the Wellcome definitions of kwashiorkor and marasmus. Clearly, the Wellcome classification is useful, among other things, for studies of the pathogenesis of oedema whereas it is misleading if used for studies of the pathogenesis of fatty liver.

The rate of clearance of the fat in the liver was remarkably slow, with up to one quarter of children not apparently metabolising any of the fat in their livers during recovery. Our figure of $1.4 \%$ /day is in broad agreement with Waterlow's two estimates of $5.5 \% /$ day and $0.9 \% /$ day, using different assumptions. If a $7 \mathrm{~kg}$ child has a $400 \mathrm{~g}$ liver, with $35 \%$ wet weight as fat $(20 \mathrm{~g}$ hepatic fat $/ \mathrm{kg}$ ) and he mobilises $1 \cdot 4 \% /$ day then only $10.6 \mathrm{~kJ} / \mathrm{kg} /$ day $(2.5 \mathrm{kcal})$ is coming from hepatic fat. Even if the higher of Waterlow's estimates is used, less than $40 \mathrm{~kJ} / \mathrm{kg} /$ day is supplied from hepatic fat. This is a relatively tiny fraction of the energy consumption of these children, who become markedly hypermetabolic during their period of rapid weight gain. ${ }^{23}$ Their dietary energy supply is closely related to their rate of recovery 2425 : the children frequently consume $800 \mathrm{~kJ} / \mathrm{kg} /$ day with $60 \%$ of this energy coming from fat; thus, children may oxidise $13 \mathrm{~g} \mathrm{fat} / \mathrm{kg} /$ day. ${ }^{25}$ Clearly, during recovery, they do not have a metabolic constraint on fat oxidation. Equally, the children increase their plasma lipoproteins within three to five days of admission to supranormal concentrations $^{26}$ : there does not seem to be a difficulty with lipoprotein synthesis during recovery. Under these circumstances, one would expect the fat in the liver to be rapidly consumed. However, our data, and those of Waterlow ${ }^{2}$ indicate that the fat is mobilised slowly. The defects that form the bases for the current theories of the pathogenesis of fatty liver in malnutrition are quickly corrected during recovery from malnutrition: they are inadequate to explain the phenomenon of persistent hepatic steatosis.

There was no overall association between liver size and liver fat despite the extensive steatosis at each stage of recovery. Our results emphasise that there is often severe fatty liver in malnutrition without significant hepatomegaly.

Ultrasound can be used non-invasively and repeatedly to assess fatty liver in further studies of malnutrition. Ethical constraints preclude the use of liver biopsy in children that are relatively well. No data exist, therefore, on the extent of fat in the liver of 'normal' and mild or moderately malnourished children in developing countries. Our results show that fatty liver occurs in undernourished children and even in one of our control children, despite normal liver function tests. It is possible that the prevalence of fatty liver in children in the developing world is greater than is generally believed.

This work was supported by the Wellcome Trust. We are grateful to Professor G Serieant, MRC Sickle Cell Laboratories, University of the West Indies for allowing us access to the ultrasound machine and to Dr H Mohammed for his help. Drs $\mathrm{R}$ Wilkes and D T Simeon gave valuable statistical advice.

Williams CD. Kwashiorkor: a nutritional disease of children associated with a maize diet. Lancet 1935;ii:1151-2.

2 Waterlow JC. Amount and rate of disappearance of liver fat in malnourished infants in Jamaica. Am f Clin Nutr 1975;28: $1330-6$

3 Garrow JS, Pike MC. The short-term prognosis of severe primary infantile malnutrition. Brf Nutr 1967;21:155-65.

McLean AEM. Hepatic failure in malnutrition. Lancet 1962; ii: $1292-4$.

5 Suskind D, Murthy KK, Suskind RM. The malnourished child: an overview. In: Suskind $R$, Lewinter-Suskind $L$ child: an overview. In: Suskind R, Lewinter-Suskind L, eds. The $1-22$.

6 Alpers DH, Isselbacker KJ. Fatty liver: biochemical and clinical aspects. In: Schiff $\mathrm{L}$, ed. Diseases of the liver. Philadelphia: J B Lippincott, 1975:815-32.

7 Jelliffe DB. Protein-calorie malnutrition in tropical preschool children (a review of present knowledge). $\mathcal{F}$ Pediatr 1959 54:227-56

8 Fletcher K. Observations on the origin of liver fat in infantile malnutrition. Am $\mathcal{F}$ Clin Nutr 1966;19:170-4.

9 McLaren DS, Faris R, Zekian B. The liver during recovery from protein-calorie malnutrition. F Trop Med Hyg 1968 , 71:271-81.

10 Saverymuttu SH, Joseph AEA, Maxwell JD. Ultrasound scanning in the detection of hepatic fibrosis and steatosis. BM7 1986;292:13-5.

11 Anonymous. Classification of infantile malnutrition. Lancet 1970;ii:302-3.

12 Jackson AA, Golden MHN. Severe malnutrition. In: Weatherall DJ, Ledington JGG, Warrell DA, eds. The Oxford textbook of medicine. Oxford: Oxford University Oxford textbook of

13 Ashworth A. Practical aspects of dietary management during rehabilitation from severe protein-energy malnutrition. rehabilitation from severe protein-energ

14 McLain DH. Drawing contours from data points. Computer fournal 1974;17:318-24.

15 Cleveland WS. Robust, locally weighted regression and smoothing scatterplots. Fournal of the American Statistical Association 1979;74:829-36. 
16 Gillman J, Gilbert C. Fatty liver of endocrine origin with special reference to fatty liver of malnourished African infants. BMF 1958;i:57-63.

17 Lewis B, Hansen JDL, Wittmann W, Krut LH, Stewart F. Plasma free fatty acids in kwashiorkor and the pathogenesis of the fatty liver. Am $\mathcal{F}$ Clin Nutr 1964;15:161-8.

18 Flores H, Seakins A, Brooke OG, Waterlow JC. Serum and liver triglycerides in malnourished Jamaican children with

19 Dhansay MA,' Spinnler Benade AJ, Donald PR. Plasma lecithin-cholesterol acyl-transferase activity and plasma lipoprotein composition and concentrations in kwashiorkor Am f Clin Nutr 1991;53:512-9.

20 Agbedana EO, Johnson AO, Taylor GO. Studies on hepatic and extrahepatic lipoprotein lipases in protein-calorie malnutrition. Am f Clin Nutr 1979;32:292-8.
21 Doherty JF, Golden MHN, Brooks SEH. Peroxisomes and the fatty liver of kwashiorkor-an hypothesis. Am $\mathcal{J}$ Clin Nutr 1991;54:674-7.

22 Cook GC. The liver after kwashiorkor. BMf 1967;iii:454-7.

23 Ashworth A. Metabolic rates during recovery from proteincalorie malnutrition: the need for a concept of specific calorie malnutrition: the need for a
dynamic action. Nature 1969;223:407-9.

24 Waterlow JC. The rate of recovery of malnourished infants in relation to the protein and calorie levels of the diet. $\mathcal{F}$ Trop Pediatr 1961;7:16-22.

25 Ashworth A, Bell R, James WPT, Waterlow JC. Calorie requirements of children recovering from protein-calorie malnutrition. Lancet 1968;ii:600-3.

26 Coward WA, Whitehead RG. Changes in serum betalipoprotein concentration during the development of kwashiorkor and in recovery. Br F Nutr 1972;27:383-94.

\section{Methotrexate for severe asthma}

Despite the major advances in the treatment of asthma that have been made in the last quarter of a century or so there are still some patients who have severe and even life threatening asthma, although receiving maximal current conventional therapy, and others who suffer the ill effects of regular high dose steroid treatment. Can anything else be done to help such patients? There have been reports of improvement in adults with severe asthma after treatment with methotrexate and a recent paper from Kansas City, Missouri (Salomon Guss and Jay Portnoy, Pediatrics 1992;89: 635-9) gives details of a small uncontrolled trial in children.

Five girls and two boys aged between 3 and 14 years were treated. All had severe asthma and were receiving regular oral prednisone in doses ranging from $30 \mathrm{mg}$ on alternate days to 60 mg daily in addition to inhaled treatment with salbutamol, sodium cromoglycate, and beclomethasone, and oral theophylline. Two of the seven patients had frequent emergency department visits and hospital admissions but one of them was thought to be noncompliant with treatment. Methotrexate was given in a starting dose of $2.5 \mathrm{mg}$ once a week and the dose was increased by $2.5 \mathrm{mg}$ every four weeks to a maximum of $17.5 \mathrm{mg}$ in two patients, 12.5 $\mathrm{mg}$ in three, and $7.5 \mathrm{mg}$ in one. One patient was unable to tolerate the drug and had only two doses.

No patient showed any improvement in the asthma in the first three months of methotrexate treatment but eventually three patients came off steroids and two were able to take a much reduced dose. Data are provided about spirometric measurements before and during treatment but they are difficult to interpret because we are not told when the values given were obtained and whether they represent an average over a period of time or single recordings. One patient showed no response after treatment for six months. One 9 year old had all her treatment stopped by her family when her asthma improved after four months of methotrexate. Two children apparently deteriorated and improved again when the course of methotrexate was interrupted and restarted.

The authors try very hard, in my opinion too hard, to make a case for methotrexate. They emphasise the toxicity of steroids in comparison with methotrexate in a quite arbitrary fashion and their assessment of their results does not seem to be totally unbiased. For instance they claim that methotrexate led to fewer emergency visits and admissions to hospital when it is quite clear from their data that there was no significant effect in these respects. They point out in high falutin terms that the study was uncontrolled and therefore the value of the drug remains uncertain but they encourage doctors to try the drug on a 'give it and see' basis. There is serious concern about the potential toxicity of methotrexate with pneumonitis, pulmonary fibrosis, and hepatic fibrosis being the main worries. In the present state of knowledge it seems to me that to encourage anything other than a strictly monitored controlled trial would be hard to justify. As far as treating individual patients is concerned, if there is a flag indicating 'proceed with extreme caution' it would seem appropriate to wave it vigorously at this point. 